\title{
Load Balancing in Mobility-on-Demand Systems: Reallocation Via Parametric Control Using Concurrent Estimation
}

\author{
Rebecca M. A. Swaszek ${ }^{1}$ and Christos G. Cassandras ${ }^{1,2}$ \\ ${ }^{1}$ Division of Systems Engineering, ${ }^{2}$ Department of Electrical and Computer Engineering \\ Boston University, Boston, MA 02215, USA \\ E-mail: $\{$ swas zek, cgc $\} @$ @bu .edu
}

\begin{abstract}
Mobility-on-Demand (MoD) systems require load balancing to maintain consistent service across regions with uneven demand subject to time-varying traffic conditions. The load-balancing objective is to jointly minimize the fraction of lost user requests due to vehicle unavailability and the fraction of time when vehicles drive empty during load balancing operations. In order to bypass the intractability of a globally optimal solution to this stochastic dynamic optimization problem, we propose a parametric threshold-based control driven by the known relative abundance of vehicles available in and en route to each region. This is still a difficult parametric optimization problem for which one often resorts to trial-and-error methods where multiple sample paths are generated through simulation or from actual data under different parameter settings. In contrast, this paper utilizes concurrent estimation methods to simultaneously construct multiple sample paths from a single nominal sample path. The performance of the parametric controller for intermediate size systems is compared to that of a simpler single-parameter controller, a state-blind static controller, a policy of no control, and a theoretically-derived lower bound. Simulation results show the value of state information in improving performance.
\end{abstract}

\section{INTRODUCTION}

Mobility-on-Demand (MoD) systems, such as those operated by ride-sharing companies Uber and Lyft, as well as traditional taxi-cab services, are comprised of a fleet of vehicles, service regions, and users who wish to travel among or within regions according to dynamic temporal-spatial demand patterns. Current research promises the advent of fully autonomous vehicles and Autonomous Taxis (ATs) which are expected to make forays into the MoD sector [1]. Service providers of these AT fleets will face myriad regulatory, safety, and management challenges. The literature surrounding the fleet management of MoD systems considers how to efficiently route vehicles among regions to meet current and projected demand, determine the appropriate fleet size, and guarantee certain levels of system performance, i.e., average wait times, availability, etc. The load balancing portion of fleet management can be either proactive in anticipating future demand or reactive in meeting current demand.

In this paper, we focus on a crucial operational challenge, that of proactive load balancing. This refers to the process

\footnotetext{
* Supported in part by NSF under grants ECCS-1509084, DMS-1664644, CNS-1645681, by AFOSR under grant FA9550-15-1-0471, by ARPA-E's NEXTCAR program under grant de-ar0000796 and by the MathWorks.
}

of dynamically redistributing the fleet so as to maintain availability across service areas to meet future predicted demand. Clearly, there is a trade-off between satisfying system-wide customer requests and driving hours logged by empty vehicles while performing load balancing operations. Whereas profit steers the driver-centric actions of traditional taxi or Uber drivers, ATs will operate cohesively under a centralized controller. Rather than many vehicles competing for users at high-demand areas (e.g., restaurant districts), AT fleet operators may spread their available vehicles across the broader service area.

Within proactive rebalancing there are two general control approaches: a set of stationary controls based upon historical data and dynamic control based upon the current state of the system. Reported research results often demonstrate their control policies using simulation with system parameters drawn from actual transportation data (taxi, commuter questionnaires) [2],[3],[4],[5],[6]. A common abstraction is to amalgamate demands from a region to a single point and utilize queueing structures to represent available vehicles or waiting customers.

A closed Jackson queueing network model with regards to the vehicles is used in [2] and the proposed controller redistributes ATs by creating a "false" user demand rate to force empty ATs from popular destination regions to popular origin regions to mitigate demand differences. This control is static in the sense that the "false" user rates are set at the beginning of an operational period and do not change with the state of the system, i.e., the varying availability of ATs among regions. To address this limitation, a time-driven (as opposed to event-driven) controller is proposed, which evenly redistributes the AT fleet among all regions at regular time intervals. Likewise, a MoD system is modeled in [5] as a closed Jackson network with static "false" user rates to redistribute vehicles and explores the trade-off between fleet size and user satisfaction as defined by average waiting time.

The receding horizon controller introduced in [7] dynamically adjusts false" user rates according to the state of the system. Greedy state-based controllers are developed in [8] to relocate available vehicles by considering the relative abundance of vehicles and waiting/expected customers in adjacent regions.

Other authors utilize a flow abstraction such that user demand rates induce vehicle "flows" between regions. In 
order to stop some regions from running out while others amass flow, a set of static controlled rates is proposed in [9] to redistribute the flow to ensure stability; [6] builds off the aforementioned work to minimize total system cost in terms of capital costs, operating costs, and passenger experience, i.e., wait time.

The literature on reactive fleet rebalancing focuses primarily on the vehicle-user assignment problem with an objective to minimize required fleet size. Whereas in proactive rebalancing, available vehicles are sent empty to other regions, in reactive rebalancing free vehicles sit idle at their last destination [10]. Ride-sharing focused papers [3],[4],[11] propose using ATs to operate carpools of varying sizes and delay times.

In this paper, we use a queueing network model akin to that in [2] in which ATs are discrete entities (as opposed to flow approximations) with an objective of minimizing the fraction of user requests that are dropped due to AT unavailability and the fraction of time ATs spend on load balancing operations driving empty between regions. The optimal control over an infinite horizon for such a system can be determined using dynamic programming, but the well-known "curse of dimensionality" renders such solutions intractable for all but very small systems.

Our approach is to transform this intractable dynamic optimization problem into a still difficult but much more manageable parametric optimization problem where the parameters are thresholds in controllers that direct redistribution based upon the relative quantity of available ATs in and en route to each region. These thresholds may be tuned to various demand patterns (rush-hour, high traffic, etc.). We propose two controllers: a time-driven single-parameter controller and an event-driven multi-parameter controller; the latter demonstrates superior performance but its parameters require more effort to tune.

The contribution of the paper is to solve the load balancing problem of a MoD system by formulating a thresholdbased parametric optimization problem and using concurrent estimation methods [12],[13] to estimate the optimal thresholds from a single observed sample path of the queueing network, thus bypassing the need for repeated trial-anderror. In addition, we derive a lower bound to assess the performance of our proposed threshold-based control.

Section II introduces the MoD system model framework while Section III details the challenges associated with finding an optimal control. Section IV] proposes two parametric threshold-based controllers. Section $\mathrm{V}$ outlines the Concurrent Estimation techniques of the Standard Clock method as well as the variation used to simulate the AT system. Section VI derives an average best possible performance lower bound and the parametric controllers' performance is demonstrated in Section VII for a 6-region example. Finally, Section VIII concludes and highlights potential future work.

\section{System Model}

We model a MoD system as a closed Jackson queueing network of $N$ nodes $\mathcal{N}=\{1, \ldots, N\}$ and $m$ resources representing regions and vehicles, respectively, similar to the model in [2]. We focus on the load balancing of an urban autonomous taxi fleet and as such shall refer to vehicles and demands as ATs and requests, respectively.

Figure 1 shows a region $i$ which consists of a queue of available ATs. In order to capture time-varying operating demand, routing and service characteristics, we divide a finite time period $[0, T]$ into $K$ intervals indexed by $k=$ $1,2, \ldots, K$, each of length $I$. Thus, the user request rate $\lambda_{i, k}$ depends on interval $k=1,2, \ldots, K$. When a user request occurs, if there is an available idle AT, then the (user, AT) pair, denoted by $\times$ and $\square$, respectively, are joined and routed with probability $p_{i, j, k}$ (including intra-region trips $i=j$ ) to an infinite-capacity server $W_{i, j}$ with service rate $\mu_{i, j, k}$. This server captures the delay experienced by users as they travel from $i$ to $j$. Upon arrival at region $j$, the pair is separated: the AT is routed with probability 1 to the idle AT queue in region $j$ and the user exits the system. If there are no available ATs at the time of the user request event, this user immediately exits the system and incurs a cost. A load balancing controller at each region, marked by $\diamond$, routes (according to some control policy) an empty available AT to server $W_{i, j}$ ultimately destined for region $j$.

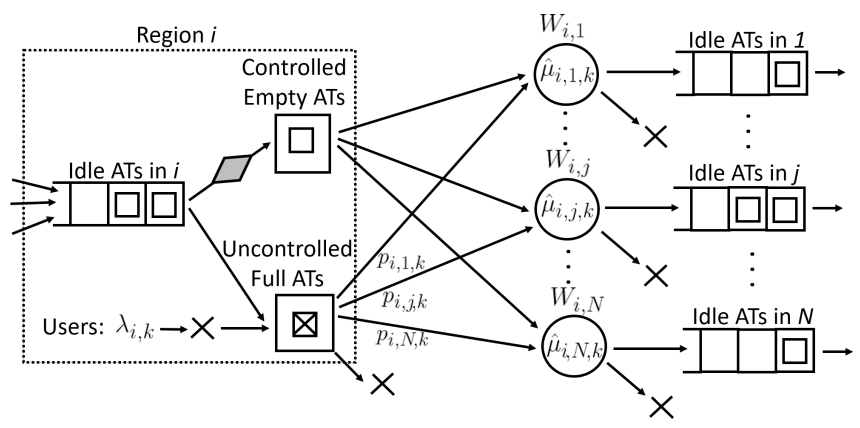

Fig. 1. Region $i$ consists of users and ATs that are coupled and routed with probability $p_{i, j, k}$ to a infinite capacity server $W_{i, j}$, then uncoupled and the AT routed into region $j$. Idle ATs may also be forced by a decision $\diamond$ to depart empty from the idle queue in $i$ for another region $j$.

\section{A. State Space}

Let $x_{i}(t) \in\{0,1, \ldots, m\}$ be the number of available idle ATs in region $i \in \mathcal{N}$ and $\boldsymbol{x}(t)=\left[x_{1}(t), \ldots, x_{N}(t)\right]$ be the idle AT vector. Let $y_{i, j}(t)$ be the number of full ATs (with passengers) en route from $i$ to $j$ and $\mathbf{Y}(t)$ be the corresponding $N \times N$ matrix populated by $y_{i, j}(t)$. Likewise, let $z_{i, j}(t)$ be the number of empty ATs in server $W_{i, j}$ and $\mathbf{Z}(t)$ be the corresponding $N \times N$ matrix populated by $z_{i, j}(t)$. Finally let $k(t) \in\{1, \ldots, K\}$ be the interval that specifies the user arrival rates $\lambda_{i, k}$, routing probabilities $p_{i, j, k}$, and service rates $\mu_{i, j, k}$ in effect at time $t$. Thus, the state of the system is $\mathscr{X}(t)=[\mathbf{x}(t), \mathbf{Y}(t), \mathbf{Z}(t), k(t)]$.

\section{B. Events}

The system dynamics are event-driven with the event set $E=E_{U} \cup E_{C}$ where $E_{U}$ and $E_{C}$ contain the uncontrollable and controllable events, respectively. We define the following uncontrollable event types within $E_{U}$ : 
- $\kappa_{k}$ event: the start of the $k$ th interval which prompts a change of $\lambda_{i, k}, p_{i, j, k}$, and $\mu_{i, j, k}$.

- $\delta_{i, j}$ event: a user request occurs for a trip departing from region $i$ with destination $j$ (with the possibility that $j=i$ ). Note that this request event does not necessitate an AT departure in the case that region $i$ has no available ATs (see Section II-D).

- $\alpha_{i, j}$ event: a full AT originating from region $i$ arrives at region $j$.

- $\nu_{i, j}$ event: an empty AT originating from region $i$ arrives at region $j \neq i$.

We also define the following controllable event types within $E_{C}$ :

- $\omega_{i, j}$ event: an empty AT (without passengers) departs from the idle AT queue in region $i$ destined for region $j \neq i$.

- $\sigma$ event: a timeout event used for time-driven control.

The control policy we select determines when controllable events are triggered. For example, a controllable $\omega_{i, j}$ event may be triggered by a timeout or the occurrence of an uncontrollable event resulting in the state of the system meeting certain criteria.

For a sample path of length $T$ of the MoD system let $\mathbf{e}=\left\{e_{1}, \ldots, e_{Q_{T}}\right\}$ be the observed event sequence, $e_{i} \in E$, with corresponding event times $\tau=\left\{\tau_{1}, \ldots, \tau_{Q_{T}}\right\}$ for a total of $Q_{T}$ events in $[0, T]$. Only at these event times $\tau_{q}$ may the state of the system change. We may now write the state of the system at time $\tau_{q}$ as $\mathscr{X}\left(\tau_{q}\right) \equiv \mathscr{X}_{q}$, with $x_{i}\left(\tau_{q}\right) \equiv x_{i, q}$, $y_{i, j}\left(\tau_{q}\right) \equiv y_{i, j, q}, z_{i, j}\left(\tau_{q}\right) \equiv x_{i, j, q}$, for all $i \in \mathcal{N}$, where $q=1, \ldots, Q_{T}$ is the asynchronous event counter.

\section{Controls}

A control action in this system consists of forcing an idle AT in some region $i$ to travel empty to some other region $j \neq i$. This action depends upon the availability of idle ATs. Let $u_{i, j, q}\left(x_{i, q}\right) \in\left\{0,1, \ldots, x_{i, q}\right\}$ be the number of empty ATs forced from $i$ to $j$ when the $q$ th event occurs and let $\mathbf{U}_{q}\left(\mathbf{x}_{q}\right)$ be the $N \times N$ matrix populated by $u_{i, j, q}\left(x_{i, q}\right)$. The following feasibility constraint is required for any control matrix $\mathbf{U}_{q}\left(\mathbf{x}_{q}\right)$ :

$$
\sum_{j=1}^{N} u_{i, j, q}\left(x_{i, q}\right) \leq x_{i, q} \quad \forall i \in \mathcal{N}
$$

For simplicity of notation, let us drop the explicit control dependence on $x_{i, q}$ and write $u_{i, j, q}$. Note that such controls may be event-driven (deployed when the state of the system satisfies certain conditions) or time-driven via the controllable timeout event $\sigma$.

\section{State Dynamics}

The inventory $x_{i, q}$ of the idle AT queue in region $i$ depends on both the uncontrollable and controllable events:

$$
x_{i, q}= \begin{cases}x_{i, q-1}+1 & e_{q}=\alpha_{j, i} \text { or } e_{q}=\nu_{j, i} \\ \max \left\{x_{i, q-1}-1,0\right\} & e_{q}=\delta_{i, j} \\ x_{i, q-1}-u_{i, j, q} & e_{q}=\omega_{i, j} \\ x_{i, q-1} & \text { otherwise }\end{cases}
$$

where $i, j \in \mathcal{N}$. Note that the max operation prevents the idle AT queue inventory from falling below 0 in the case that a $\delta_{i, j}$ user request event occurs and there are no idle ATs, i.e., when a user exits the system prematurely as in Figure 1.

The number of full ATs $y_{i, j, q}$ en route from region $i$ to region $j$ evolves according to:

$$
y_{i, j, q}= \begin{cases}y_{i, j, q-1}+1 & e_{q}=\delta_{i, j} \text { and } x_{i, q-1}>0 \\ y_{i, j, q-1}-1 & e_{q}=\alpha_{i, j} \\ y_{i, j, q-1} & \text { otherwise }\end{cases}
$$

Likewise the number of empty ATs en route from $i$ to $j$ evolves according to:

$$
z_{i, j, q}= \begin{cases}z_{i, j, q-1}+u_{i, j, q} & e_{q}=\omega_{i, j} \\ z_{i, j, q-1}-1 & e_{q}=\nu_{i, j} \\ z_{i, j, q-1} & \text { otherwise }\end{cases}
$$

At time instants $t=k \mathcal{I}, k \in\{1, \ldots, K\}$ the interval index $k$ changes upon the occurrence of a $\kappa_{k}$ event, thus causing the time-varying parameters to change for all regions: $k_{q}=$ $k_{q-1}+1$

\section{E. Objective Function}

The primary concern in the relevant literature of MoD systems is to meet all or most of the user requests while also minimizing load balancing operations. We formalize this trade-off in an objective function which minimizes a weighted sum of the probability that a user's request does not result in an AT departure (i.e., is rejected as in Figure 1) and the probability that an AT is driving empty performing load balancing operations. The evaluation of these probabilities is generally infeasible because of the fast growth of the state space, rendering this task intracatable except for the simplest of systems. In order to assess the effect of controls $u_{i, j, q}$ on system performance, we replace these probabilities with estimates consisting of the fraction of rejected requests and total time spent driving empty in a sample path over $[0, T]$. Let $\boldsymbol{\rho}_{T}^{i}=\left\{\rho_{i, 1}, \ldots, \rho_{i, D_{T}^{i}}\right\}$ be the event times of all $\delta_{i, j}, j \in \mathcal{N}$ user request events at region $i$ where $D_{T}^{i}$ is the total observed number of such events, and let $\mathbf{1}[\cdot]$ be the usual indicator function. As defined in Section II-B, there are a total of $Q_{T}$ events in $[0, T]$ and the $q$ th event occurs at time $\tau_{q}$.

The objective function we define is:

$$
\begin{aligned}
J\left(\mathscr{X}_{0}\right) & =E\left[w \frac{\sum_{i=1}^{N} \sum_{q=1}^{D_{T}^{i}} \mathbf{1}\left[x_{i}\left(\rho_{i, q}\right)=0\right]}{\sum_{i=1}^{N} D_{T}^{i}}\right. \\
+(1-w) & \left.\frac{\sum_{q=1}^{Q_{T}} \sum_{i=1}^{N} \sum_{j=1}^{N}\left(\tau_{q}-\tau_{q-1}\right) z_{i, j, q}}{T m}\right]
\end{aligned}
$$

where $w \in(0,1]$ is a weight coefficient. The first part of 5 refers to the fraction of rejected users unable to obtain an available idle AT (the numerator is the total number of all user request events). The second part refers to the fraction of vehicle-hours when ATs drive empty (the denominator is the total vehicle-hours driven by the $m$ vehicles in the fleet over 
$[0, T])$. The weight coefficient $w$ is used to quantify the tradeoff between customer satisfaction and load balancing effort. We exclude $w=0$ as the optimal control is trivial when customer satisfaction is irrelevant. If $w=1$, the optimal control is still difficult to determine: although load balancing may not be a direct cost, the unavailability of ATs while performing load balancing operations creates an indirect cost. Note that the objective function in (5) is properly normalized so as to give values bounded by $[0,1]$ and the weight $w$ creates a convex combination of the two objective components.

The optimization problem we formulate based on (5) is to determine a control policy $\mathbf{U}(\mathscr{X})$ so as to minimize this objective:

$$
J^{*}\left(\mathscr{X}_{0}\right)=\min _{\mathbf{U}(\mathscr{X})} J\left(\mathscr{X}_{0}\right)
$$

\section{Optimal Control Policy}

Let us first consider a simpler version of the problem where we assume that user arrivals occur according to a Poisson process with fixed rate $\lambda_{i}$ and that each infinitecapacity server $W_{i, j}$ has exponentially distributed service times with mean service time $\frac{1}{\mu_{i, j}}$. Thus, the MoD system is described by a finite-dimensional continuous time Markov chain. As an example, the simplest possible such system corresponds to $N=2$ and $m=1$, in which case the single AT is in one of eight possible states: idle in either region 1 or 2 , en route with a passenger in one of the four infinite capacity queues $W_{1,1}, W_{1,2}, W_{2,1}, W_{2,2}$ or en route empty performing load balancing in $W_{1,2}^{E}, W_{2,1}^{E}$ (with the subscript $E$ denoting "empty"). For this simple system, the optimal control policy may be found analytically as shown in Appendix A and B. For any larger system, we turn to dynamic programming (DP) [14] to determine the optimal control policy for the average cost over the infinite horizon. The cost $C(\mathscr{X})$ associated with being in state $\mathscr{X}=[\mathbf{x}, \mathbf{Y}, \mathbf{Z}]$ is as follows:

$$
\begin{aligned}
C(\mathscr{X})=w \sum_{i=1}^{N} \frac{\sum_{j=1}^{N} \lambda_{i, j} \mathbf{1}\left[x_{i}=0\right]}{\sum_{i=1}^{N} \sum_{j=1}^{N}\left(\lambda_{i, j}+\mu_{i, j}\right)} \\
+(1-w) \frac{\sum_{i=1}^{N} \sum_{j=1}^{N} z_{i, j}}{m}
\end{aligned}
$$

Let $S$ and $U$ denote the state and control spaces, respectively, let $\bar{g}(i, u)$ be the cost of state $i$ as defined by (7), and suppose that the state space cardinality is $|S|=n$. Let $P_{i, j}(u)$ be the transition probability from state $i$ to state $j$ under control $u$. We wish to solve for the optimal cost function $J^{*}$ which satisfies for all $i \in S$ the deterministic form of Bellman's equation [14]:

$$
J^{*}(i)=\min _{u \in U(i)} \bar{g}(i, u)+\sum_{j=1}^{n} P_{i, j}(u) J^{*}(j)
$$

Linear programming or policy iteration may be used to solve (8). In the linear programming formulation, we use the differential cost vector $[h(1), \ldots h(n)]$ (necessary in the absence of discounting) and the optimal cost $\bar{J}$ as the decision variables [14]:

$$
\begin{gathered}
\max _{\bar{J}, h(1), \ldots h(n)} \bar{J} \quad \text { s. t. } \\
\bar{J}+h(i) \leq \bar{g}(i, u)+\sum_{j=1}^{n} P_{i, j}(u) h(j) \quad \forall i \in S, u \in U(i)
\end{gathered}
$$

The resulting optimal steady-state distribution is given by the Lagrange multipliers of $[h(1), \ldots, h(n)]$ and the binding constraints indicate optimal policies. As an example, the full linear programming problem for the $N=2, m=1$ system may be found in Appendix C.

Likewise, the policy iteration method solves a linear system of equations $\mathbf{h}_{\mu}+\mathbf{e} \bar{J}_{\mu}=\overline{\mathbf{g}}_{\mu}+\mathbf{P}_{\mu} \mathbf{h}_{\mu}$ in which $\mathbf{h}_{\mu}$ and $\overline{\mathbf{g}}_{\mu}$ are the $n \times 1$ vectors of $\bar{g}(i)$ and $h(i)$ under some policy $\mu$, $\mathbf{P}_{\mu}$ is the transition probability matrix populated by $P_{i, j}(\mu)$, and $\mathbf{e}$ is a $n \times 1$ vector with $e_{i}=1$ for all $i=1, \ldots, n$. Policy iteration is the more efficient method to find the optimal control as it solves smaller linear programs and requires less memory, although convergence to the optimal policy may be slow, depending on the initial policies chosen.

However, both methods are limited to small systems as the state space grows combinatorially with the number of regions and ATs. For a system with $N$ regions and $m$ ATs, the cardinality of the state space is $\left(\begin{array}{c}m+2 N^{2}-1 \\ 2 N^{2}-1\end{array}\right)$ and the cardinality of states with at least $N-1$ controls is: $\left(\begin{array}{c}m+2 N^{2}-1 \\ 2 N^{2}-1\end{array}\right)-\left(\begin{array}{c}m+2 N^{2}-N-1 \\ 2 N^{2}-N-1\end{array}\right)$. For example, an AT system with $N=6$ regions and $m=50$ taxis has over $3 \times 10^{34}$ states. Utilizing sparse matrices and shared cloud computing facilities, the largest possible systems that we have been able to analyze using the policy iteration method consists of $N=2$ regions and $m=10$ ATs and of $N=3$ regions and $m=5$ ATs. The latter required only 31 GB of memory, while solving for $N=3$ regions and $m=6$ ATs required more than the $256 \mathrm{~GB}$ allotted to a full node of 28 cores. This provides the motivation for seeking alternative control policies and assessing their performance using the fraction estimates in (5).

\section{Parametric Control Policies}

In this section, we introduce a parametric controller for larger MoDs expanding upon the framework of the "realtime" controller in [2]; this "real-time" controller rebalances ATs evenly among regions every half hour using an integer linear programming approach to minimize the expected load balancing time. Unlike our loss model in which user requests may be rejected if there are no available ATs, the model in [2] includes a queue for waiting users such that the number of ATs associated with a region is the sum of available ATs and ATs en route with the number of users queued up waiting for an AT subtracted from the latter.

Let $\Theta=\left[\theta_{1}, \ldots, \theta_{N}\right]$ be a parameter vector with $\theta_{i} \geq 0$, $i=1, \ldots, N$ and $\sum_{i=1}^{N} \theta_{i} \leq m$. This vector defines a "fill to" level for each of the $N$ regions; this is akin to $(s, S)$ threshold policies in supply chain and inventory management [14] where $s$ is a "fill to" level such that when an inventory 
drops below it, a supply request is triggered (similarly, crossing $S$ from below triggers a request to stop the supply process). Note that each interval $k=1,2, \ldots, K$ may have its own set of parameters to account for different request patterns and traffic conditions. For simplicity of notation, let $a_{i}(t)$ be the total number of ATs available at or en route to region $i$ at time $t$ :

$$
a_{i}(t)=x_{i}(t)+\sum_{j=1}^{N}\left(y_{j, i}(t)+z_{j, i}(t)\right)
$$

Furthermore let us define a quantity $D_{i}(t)$ that is the supply of available excess ATs if positive or the demand for ATs if negative in region $i: \quad D_{i}(t)=\min \left\{a_{i}(t)-\theta_{i}, x_{i}(t)\right\}$ Note that $D_{i}(t)$ is an integer quantity as $\theta_{i}, a_{i}(t)$, and $x_{i}(t)$ are all integers. In order for feasible AT redistribution actions to be triggered, it is a necessary condition that the overall supply must exceed the demand in the following inequality:

$$
\sum_{k \in\left\{i \in \mathcal{N} \mid D_{i}(t)>0\right\}} D_{k}(t) \geq \sum_{j \in\left\{i \in \mathcal{N} \mid D_{i}(t) \leq 0\right\}} D_{j}(t)
$$

This simply asserts that there is an adequate number of available ATs in regions which are above their "fill-to" levels specified in $\Theta$ which can be used to supply those regions whose queues of available ATs are below their "fill-to"levels.

Assuming for the moment that there exists a well-defined mechanism for triggering a process to redistribute ATs among regions (further discussed in Sections IV-A and IV-B, this process consists of the following integer linear program with decision variables $u_{i, j} \in\{0,1,2, \ldots\}$ :

$$
\begin{gathered}
\min _{u_{i, j}, j, j \in \mathcal{N}} \sum_{i=1}^{N} \sum_{j=1}^{N} \frac{u_{i, j}}{\mu_{i, j}} \\
\text { s. t. } \theta_{i} \leq a_{i}(t)+\sum_{j=1}^{N}\left(u_{j, i}-u_{i, j}\right) \quad i \in \mathcal{N} \\
\sum_{j=1}^{N} u_{i, j} \leq x_{i}(t) \quad i \in \mathcal{N} \\
u_{i, j} \in\{0,1,2, \ldots\} \quad i, j \in \mathcal{N}
\end{gathered}
$$

The objective function of (12) minimizes empty vehicle driving time; the first constraint requires the intended inventory for each region to meet or exceed "fill to" levels $\theta_{1}, \ldots, \theta_{N}$, and the second constraint maintains feasibility.

In order to bypass the difficulty of integer programming, we rewrite 12 as a relaxed linear program in the form of a minimum cost flow problem in which regions indexed by $i$ with positive supply $D_{i}(t)$ are sources and negative demand $D_{i}(t)$ are sinks.

$$
\begin{gathered}
\min _{u_{i, j}, i, j \in \mathcal{N}} \sum_{i=1}^{N} \sum_{j=1}^{N} \frac{u_{i, j}}{\mu_{i, j}} \\
\text { s. t. } D_{i}(t) \geq \sum_{j=1}^{N}\left(u_{i, j}-u_{j, i}\right) \quad i \in \mathcal{N} \\
u_{i, j} \geq 0 \quad i, j \in \mathcal{N}
\end{gathered}
$$

Note that the single constraint in (13) encompasses both constraints in (12): $(i)$, if $D_{i}(t)=a_{i}(t)-\theta_{i}$, the constraint in (13) is identical to the first constraint of (12); (ii) if $D_{i}(t)=x_{i}(t)$, then $i$ is a source such that no flow is directed to it, i.e., $\sum_{j=1}^{N} u_{j, i}=0$, therefore, the constraint in (13) becomes identical to the second constraint of (12). We recover the integer solution to (12) from this linear program since integer solutions are a property of minimum cost flow linear programs with integer sink and source quantities [15].

A potential problem associated with this policy is that, depending upon the frequency at which these control actions are implemented, there could be empty ATs cycling inefficiently such that $z_{i, j}(t)>0$ and $z_{j, i}(t)>0$ simultaneously. To address this issue, we introduce an additional parameter $\Omega$ which acts as either a scalar "timeout" in a time-driven controller in Section IV-A or as an integer threshold in an event-driven controller in Section IV-B In either case, the role of $\Omega$ is to define the mechanism that triggers the process of solving 13 so as to determine the values of the control variables $u_{i, j}, i, j \in \mathcal{N}$.

\section{A. Single Scalar Parameter Time-Driven Controller}

For a time-driven controller, let the scalar parameter $\Omega \in$ $(0, \infty)$ be associated with the timeout event $\sigma$ we defined in Section II-B such that event $\sigma$ triggers the control actions in (13) every $\Omega$ time units. The "real-time" controller proposed in [2] can be recovered from our controller by setting all $\theta_{i}=\left\lfloor\frac{m}{N}\right\rfloor, i \in \mathcal{N}$ in $(12)$ and $\Omega=30$ minutes. This timedriven single scalar parameter controller is quite effective, but does not account for the system-specific demand rates. For example consider a heterogeneous two-region system this control of distributing the fleet equally between them would perform badly if one region experienced far greater requests than the other.

\section{B. $N+1$ Integer Parameter Event-Driven Controller}

In order to avoid the aforementioned inefficiencies associated with equally spreading out the fleet among regions of dissimilar demand, we define an event-driven controller to trigger a solution of problem 13 whenever $a_{i}(t)$, the number of ATs in or en route to a region, drops below the threshold $\theta_{i}$ by some amount. In the simplest case, this occurs as soon as $a_{i}(t)<\theta_{i}$, corresponding to a greedy mechanism that pulls a single empty AT from the nearest region $j$ with $a_{j}(t)>\theta_{j}$ and setting $u_{i, j}=1$. However, since we have at our disposal a central controller with full information of all region states and AT locations, we can do better than that as explained next.

Let us redefine $\Omega \in\{1,2, \ldots, m\}$ as an integer-valued threshold parameter used to trigger the control actions resulting from a solution of (13) whenever the condition $\theta_{i}-a_{i}(t)>\Omega$ is satisfied. This will effectively send a total of $\theta_{i}-a_{i}(t)$ empty ATs to region $i$. However, this is still a region-specific control that may be inefficient over the system as a whole. As such, we instead trigger the control when the sum-positive difference between the "fill-to" levels $\theta_{i}$ and available or en route ATs $a_{i}(t)$ surpasses threshold $\Omega$ across 
all regions for a global centralized control policy triggered by:

$$
\sum_{j \in\left\{i \in \mathcal{N} \mid a_{i}(t)<\theta_{i}\right\}}\left(\theta_{j}-a_{j}(t)\right)>\Omega
$$

For example, consider the state of a system with $N=$ $4, m=20$ in Fig. 2 The control parameter vector $\Theta=$ $[5,3,4,5]$ is hashed in black and let $\Omega=2$. After each event, the controller checks inequalities (11) and (14); if both hold, then $\omega_{i, j}$ events are induced as per (13). In this example, (11) holds with $(0+1+0+2) \leq(3+0+3+0)$ and (14) holds with $(0+1+0+2)>2$.

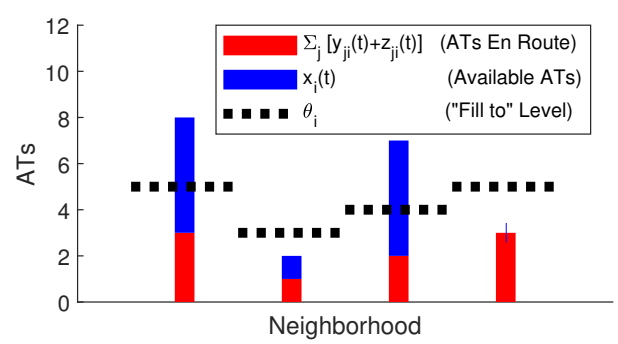

Fig. 2. Control parameters $\theta_{i}$ represent a "fill to" level for the number of ATs available at or en route to region $i$. Below this threshold represents need; above this threshold represents excess inventory to possibly send elsewhere.

This controller is triggered when one of the events defined in Section II-B causes a change in the value of some $a_{i}(t)$ in (14) so that the sum crosses $\Omega$ either from below or from above. This may happen in two ways: $(i)$ When event $\delta_{i, j}$ occurs, i.e., a user requests to go from region $i$ to $j$, which alters $x_{i}(t)$ and $y_{i, j}(t)$ as per the state dynamics (2) and (3), respectively (note that this assumes (11) already holds). (ii) Event $\alpha_{j, i}$ or $\nu_{j, i}$ occurs, i.e., a full (respectively, empty) AT arrives at region $i$ and alters the value of $x_{i}(t)$ and $y_{j, i}(t)$ (respectively, $z_{j, i}(t)$ ).

While the time-driven controller triggers control actions after a predetermined length of time regardless of the state of the system, the event-driven controller is only triggered when the inventory levels fall sufficiently low across all regions.

\section{C. $N^{2}$ Parameter Static Controller}

Both of the previously described parametric controllers require tuning the threshold parameters via simulation or through a data-driven on-line adaptation process. We shall compare in Section VII these controllers to an alternative simpler parametric controller introduced in [9] whose parameters are determined by linear programming and rely solely on the model parameters $\lambda_{i, j}$ and $\mu_{i, j}$. This time-invariant and state-blind control sends empty ATs from $i$ to $j$ at a static rate $r_{i, j}, i, j \in \mathcal{N}$ such that there are $N^{2}$ rate parameters. These static rate parameters are determined by the following linear program that minimizes empty travel time and seeks to equal the inflow and outflow of ATs at each region:

$$
\min _{r_{i, j}} \sum_{i=1}^{N} \sum_{j=1}^{N} \frac{r_{i, j}}{\mu_{i, j}}
$$

$$
\begin{array}{rlrl}
\text { s. t. } \quad \sum_{j=1}^{N}\left(\lambda_{i, j}+r_{i, j}\right) & =\sum_{j=1}^{N}\left(\lambda_{j, i}+r_{j, i}\right) & i \in \mathcal{N} \\
r_{i, j} \geq 0 & i, j \in \mathcal{N}
\end{array}
$$

This linear program is always feasible. This is easily seen by taking, for example, $r_{i, j}=\lambda_{j, i} \forall i, j \in \mathcal{N}$, which satisfies the first constraint by sending back empty taxis at the same rate and satisfies the second constraint as $\lambda_{i, j} \geq 0$. This control is state-blind since it does not depend on the current state or even the fleet size. Note that in the case that such a controller as a comparison baseline at time $t$ directs an AT to leave region $i$ for some $j$ but $x_{i}(t)=0$ (i.e., region $i$ does not have any idle ATs) neither a penalty nor an event $\omega_{i, j}$ occur. We use this state-blind controller to demonstrate the importance of a dynamic (state-driven) control strategy in the example system of Section VII

\section{Concurrent Estimation Methods}

In order to find well performing parameters for both the time-driven and event-driven controllers, we turn to concurrent estimation methods to simulate the effects of many different sets of parametric controls from a single sample path and use the resulting performances to direct the evolution of the next set of control parameters to test.

Concurrent estimation/simulation methods help in problems such as this AT fleet load balancing problem where we wish to test the performance of discrete parameters $\boldsymbol{\theta}$ whose effects on the objective function $J(\boldsymbol{\theta})$ are difficult to determine analytically. The off-line brute force approach to simulate each of the possible sets of parameters is very inefficient and time consuming. On-line each possible parameter set could be tested in a trial and error process, but this too is inefficient and may be impossible given the available time. In the time-driven control, let $\boldsymbol{\theta}=[\Omega]$ and in the event-driven control let $\boldsymbol{\theta}=\left[\theta_{1}, \ldots, \theta_{N}, \Omega\right]$.

The off-line Standard Clock (SC) method of concurrent simulation constructs sample paths from a nominal simulated sample path in order to simulate the performance $J(\boldsymbol{\theta})$ under various policies $\theta$ with the drawback of possibly simulating many fictitious events. Standard Clock is used to simulate stochastic timed automata with a Poisson clock structure such as our AT system which is an event-driven system with exponentially distributed event lifetimes. In each state $x$ there is a set of feasible events $\Gamma(x)$. Once the system is in state $x$ it stays there for a exponential amount of time with mean $\frac{1}{\Lambda(x)}$ where $\Lambda(x)=\sum_{i \in \Gamma(x)} \lambda_{i}$ and $\lambda_{i}$ is the rate at which event $i$ occurs. The distribution of the next triggering event $i$ is given by

$$
p(i, x)=\frac{\lambda_{i}}{\Lambda(x)}, i \in \Gamma(x)
$$

One may uniformize the Markov chain by introducing a uniform rate:

$$
\gamma \geq \Lambda(x)
$$

and replace $\Lambda(x)$ with this rate $\gamma$ for all states $x$ and force the additional probability flow $[\gamma-\Lambda(x)]$ to be a "fictitious" event that is a self loop back to $x$ leaving the state unchanged. 
The Standard Clock method builds off this uniformized model and chooses $\gamma=\Lambda$ with

$$
\Lambda=\sum_{i \in \mathcal{E}} \lambda_{i}
$$

where $\mathcal{E}$ is the set of all events. Note that this choice of $\gamma$ has the potential to be much larger than any $\Lambda(x)$ thus forcing the additional probability flow $[\Lambda-\Lambda(x)]$ to also be large for many states $x$. This causes many fictitious events which slow down simulations. With this new uniform rate $\gamma$ the triggering event probability is now

$$
p_{i}=\lambda_{i} / \Lambda
$$

which could potentially be much smaller than (16). The occurrence of the $k$ th event $E_{k}$ in the event set $\mathcal{E}=1, \ldots, N$ may be determined by a random number $U_{k}$ :

$$
E_{k}= \begin{cases}1 & \text { if } 0 \leq U_{k} \leq \lambda_{1} / \Lambda \\ 2 & \text { if } \lambda_{1} / \Lambda<U_{k} \leq\left(\lambda_{1}+\lambda_{2}\right) / \Lambda \\ \cdots & \\ N & \text { if }\left(\lambda_{1}+\ldots+\lambda_{N-1}\right) / \Lambda<U_{k} \leq 1\end{cases}
$$

By construction (20) allows all events to occur in any state; however, if the event $E_{k}$ is not feasible at state $x$ than it is a fictitious event i.e. a self-loop.

The following steps outline the method of constructing $M+1$ sample paths concurrently where $f_{m}(\cdot)$ is the state transition function [13]:

\section{CONSTRUCT STANDARD CLOCK}

$\left\{V_{1}, V_{2}, \ldots\right\}, V_{k} \sim 1-e^{-t}, t>0$

For every constructed sample path $m=0,1, \ldots, M$ :

2. DETERMINE TRIGGERING EVENT $E_{m}$ BY (20)

3. UPDATE STATE $X_{m}$ :

$$
X_{m}:=f_{m}\left(X_{m}, E_{m}\right)
$$

4. RESCALE INTEREVENT TIME $V$ :

$$
V_{m}=V / \Lambda_{m}
$$

Note that in Step 1 Standard Clock generates inter-event times from an exponential distribution with parameter 1 , thus for models with event rate $\Lambda \neq 1$ Step 3 adjusts the sequence $\left\{V_{1}, V_{2}, \ldots\right\}$ by rescaling $V_{k}(\Lambda)=V_{k} / \Lambda$.

\section{A. Variation of Standard Clock}

We introduce a variation on the Standard Clock method which takes advantage of models in which there is a substantial subset of events that are feasible across all states $x$ and creates fewer fictitious events with a drawback of slightly more calculations on per event.

Let $\xi \subseteq \mathcal{E}$ be the subset of the $C$ events that are feasible in every state $x$ :

$$
\xi=\{i \in \mathcal{E} \mid i \in \Gamma(x), \forall x\}
$$

and let $\xi_{x}$ be the other feasible events in state $x$ :

$$
\xi_{x}=\{i \in \mathcal{E} \mid i \in \Gamma(x), i \notin \xi\}
$$

Further, set the uniformized rate $\gamma$ as in 17

$$
\gamma=\max _{x} \Lambda(x)
$$

Similar to (20), for state $x$ the range of $U_{k}$ is partitioned to the $C$ common events and the remaining events in $\xi_{x}$ (plus fictitious events if there is any remaining range for $U_{k}$ ). Let $B$ be the probability that the event is within this common event set $\xi$ :

$$
B=\frac{\sum_{i \in \xi} \lambda_{i}}{\gamma}
$$

and let $B_{x}$ be the probability that the event is feasible in state $x$ :

$$
B_{x}=\frac{\sum_{i \in \Gamma(x)} \lambda_{i}}{\gamma}
$$

(Clearly at least one $B_{x}=1$; states with $B_{x}<1$ have the potential for fictitious events to occur.)

If $U_{k} \leq B$, then the event is in $\xi$

$$
E_{k}= \begin{cases}1 & \text { if } 0 \leq U_{k} \leq \lambda_{1} / \gamma \\ 2 & \text { if } \lambda_{1} / \Lambda<U_{k} \leq\left(\lambda_{1}+\lambda_{2}\right) / \gamma \\ \cdots & \\ C & \text { if }\left(\lambda_{1}+\ldots+\lambda_{C-1}\right) / \gamma<U_{k} \leq B\end{cases}
$$

If $U_{k}>B$, then event $E_{k}$ is either in $\xi_{x}$ or fictitious

$$
E_{k}= \begin{cases}\xi_{x}(1) & \text { if } B<U_{k} \leq B+\frac{\lambda_{\xi_{x}(1)}}{\gamma} \\ \ldots & \text { if } B+\frac{\lambda_{\xi_{x}(1)}+\ldots+\lambda_{\xi_{x}\left(\left|\xi_{x}\right|-1\right)}}{\gamma}<U_{k} \leq B_{x} \\ \xi_{x}\left(\left|\xi_{x}\right|\right) & B_{x}<U_{k} \\ \text { Fictitious } & \end{cases}
$$

Let $(21),(24)$, and $(26)$ be pre-calculated as the common events in $\xi$ will occur often while let (22), 25, and (27) be calculated on a need basis as the large state space renders them impossible to save for all states $x$.

The following four steps construct $M+1$ concurrent sample paths:

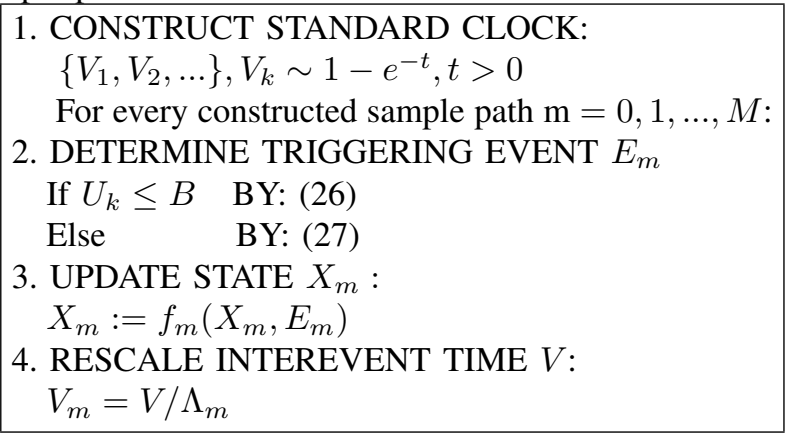

The advantage of this variation is that by setting a smaller $\gamma$ many fewer fictitious events occur at the cost of the additional calculations in (27). This method works well for our AT model as the set of common events - all user request events - is quite large: $\xi=\delta_{k, j}, k, j \in \mathcal{N}$ such that the probability $B$ of an event being within the common event set is:

$$
B=\frac{\sum_{i, j} \lambda_{i, j}}{\gamma}
$$

with $\gamma$ set as per (23) to be:

$$
\gamma=\sum_{i, j} \lambda_{i, j}+m \max _{i, j} \mu_{i, j}
$$


Table II shows the minimum and maximum percent of fictitious events possible for the Standard Clock method and variation thereof (see the example in Section VII].

TABLE I

Percent Fictitious Events

\begin{tabular}{|l|c|c|}
\cline { 2 - 3 } \multicolumn{1}{c|}{} & \multicolumn{1}{c|}{ Minimum } & Maximum \\
\hline SC & $1-\frac{\sum_{i, j} \lambda_{i, j}+m \max _{i, j} \mu_{i, j}}{\sum_{i, j}\left(\lambda_{i, j}+m \mu_{i, j}\right)}$ & $1-\frac{\sum_{i, j} \lambda_{i, j}}{\sum_{i, j}\left(\lambda_{i, j}+m \mu_{i, j}\right)}$ \\
\hline Variation & 0 & $1-\frac{\sum_{i, j} \lambda_{i, j}}{\sum_{i, j} \lambda_{i, j}+m \max _{i, j} \mu_{i, j}}$ \\
\hline
\end{tabular}

We use concurrent simulation to find well performing control parameter vectors via a greedy iterative search and a broader random search of the control space.

\section{B. Greedy Iterative Search}

In an iterative process, we find well performing control vectors by following a greedy process of following vector changes that lead to better performances. For example, consider the time-driven controller with its single parameter $\Omega$ starting at some value $\Omega^{0}$. We may use concurrent simulation to compare how the slight control deviations such as $\Omega^{0}-1$, and $\Omega^{0}+1$ perform and direct the next controller: $\Omega^{1}=$ $\operatorname{argmin}_{\Omega}\left\{J\left(\Omega^{0}-1\right), J\left(\Omega^{0}\right), J\left(\Omega^{0}+1\right)\right\}$. Iterated again and again with $\Omega^{k+1}=\operatorname{argmin}_{\Omega}\left\{J\left(\Omega^{k}-1\right), J\left(\Omega^{0}\right), J\left(\Omega^{0}+k\right)\right\}$ and with an increasing simulation length $T$, this iterative method gravitates towards well performing controls. As this requires simulation, it cannot find the optimal control, but hones in on a flat region of the objective function.

Likewise for the event-driven controller with $N+1$ control parameters, the parameter vector may start as $\left[\theta_{1}^{0}, \theta_{2}^{0}, \ldots \theta_{N}^{0}, \Omega^{0}\right]$, and the first iteration concurrently creates sample paths for the following control parameters:

$$
\begin{gathered}
{\left[\theta_{1}^{0}+1, \theta_{2}^{0}, \ldots \theta_{N}^{0}, \Omega^{0}\right]} \\
{\left[\theta_{1}^{0}, \theta_{2}^{0}+1, \ldots \theta_{N}^{0}, \Omega^{0}\right]} \\
\ldots \\
{\left[\theta_{1}^{0}, \theta_{2}^{0}, \ldots \theta_{N}^{0}+1, \Omega^{0}\right]} \\
{\left[\theta_{1}^{0}, \theta_{2}^{0}, \ldots \theta_{N}^{0}, \Omega^{0}+1\right]} \\
{\left[\theta_{1}^{0}-1, \theta_{2}^{0}, \ldots \theta_{N}^{0}, \Omega^{0}\right]} \\
{\left[\theta_{1}^{0}, \theta_{2}^{0}-1, \ldots \theta_{N}^{0}, \Omega^{0}\right]} \\
\quad \ldots \\
{\left[\theta_{1}^{0}, \theta_{2}^{0}, \ldots \theta_{N}^{0}-1, \Omega^{0}\right]} \\
{\left[\theta_{1}^{0}, \theta_{2}^{0}, \ldots \theta_{N}^{0}, \Omega^{0}-1\right]}
\end{gathered}
$$

At the end of the iteration, the next control parameter picked as the next nominal path would be that which performed the best such that each iterate follows the path of steepest descent.

\section{Random Search of the Control Space}

The greedy iterative process as described in the previous subsection searches locally within a small section of the control space and the final selected control vector, likely in a local minimum, is a function of the starting control vector $\left[\theta_{1}^{0}, \theta_{2}^{0}, \ldots \theta_{N}^{0}, \Omega^{0}\right]$.

Instead, utilizing the ability of concurrent estimation to simulate many samples paths at once, we explore more of the control space by selecting control vectors randomly. Control vectors may be selected randomly using the discrete uniform distribution: UNIFORMLY RANDOMLY SELECT A CONTROL PARAMETER VECTOR

\section{SET CONTROL VECTOR}

$$
\theta_{i} \sim \operatorname{unif}\{0, m\} \forall i \in \mathcal{N}, \Omega \sim \operatorname{unif}\{1, m\}
$$

2. DETERMINE IF CONTROL VECTOR FEASIBLE if $\sum_{0}^{N} \theta_{i}>m$ return to step 1.

in which the second step verifies that the control vector is feasible. Note that if $\Omega>\sum_{0}^{N} \theta_{i}$, the control cannot be triggered rendering a feasible but clearly useless control.

The control space of this AT load balancing problem is so large that it is not feasible to randomly simulate even a small percent of the possible controls for a long enough time to get a decent estimation of the performance. This justifies the following algorithm that finds families of well performing control vectors by iteratively shrinking the permissible control space. Instead of selecting control vectors by $\sim$ unif $\{1, m\}$, let us define an lower and upper bound $\underline{\theta_{i}}$ and $\overline{\theta_{i}}$ or $\underline{\Omega}$ and $\bar{\Omega}$. These bounds will iteratively decrease the size of the search-able control space resulting in small permissible bands for each control parameter. Let the first iteration of the algorithm start with $\underline{\theta_{i}}=0, \overline{\theta_{i}}=m \forall i \in$ $\mathcal{N}, \underline{\Omega}=1, \bar{\Omega}=m$.

Let $T$ be the length of the simulated sample paths, $L$ be the number of sample paths to concurrently simulate, and $K$ be the number of times to run a $L$ concurrent sample paths: ITERATION OF RANDOM SEARCH ALGORITHM for $k=1: K$

1. Find $L$ feasible control vectors.

for $l=1: L$

1A. Uniformly randomly select the $l$ th control vector: $\theta_{i} \sim \operatorname{unif}\left\{\theta_{i}, \overline{\theta_{i}}\right\} \forall i \in \mathcal{N}, \Omega \sim \operatorname{unif}\{\underline{\Omega}, \bar{\Omega}\}$ 1B. If $\sum_{0}^{N} \theta_{i}>\bar{m}$, return to step $1 \mathrm{~A}$.

\section{end}

2. Simulate $L$ sample paths with control vectors from step 1 for $T$ time units via concurrent estimation.

3. Choose the best performing control vector and label end it $\left[\theta_{1}^{* k}, \ldots, \theta_{N}^{* k}, \Omega^{* k}\right]$.

Redefine: $\theta_{i}=\min _{k}\left\{\theta_{i}^{* k}\right\}, \overline{\theta_{i}}=\max _{k}\left\{\theta_{i}^{* k}\right\}$,

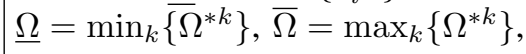

Each iteration of the above algorithm will decrease the size of the search-able control space as poorly performing control parameters are excluded in the Redefine step. This method, like the iterative search, may find areas of local minima. However, this algorithm may be run multiple times 
in independent trials to show that the final families of controls are very similar.

The choice of $K, L$, and $T$ as well as the number of iterations will determine the final size and composition of the search-able control space. $T$, the length of the simulation, must be long enough to produce decent estimations of the control performance, but for the first couple of iterations it need not be too long as these first iterations weed out the especially poor performing control parameters. As the bands of search control parameters $\left[\underline{\theta_{i}}, \overline{\theta_{i}}\right]$ thins, $T$ should increase such as to produce better estimations of system performance under the various control vectors. $L$, the number of sample paths concurrently estimated, should be large enough to render a good representation of the search space. Likewise, $K$ should be large enough that many possible control paths are simulated.

Note that we could concurrently simulate $K \times L$ sample paths and pick the best $K$ control vectors to label as $\left[\theta_{1}^{* k}, \ldots, \theta_{N}^{* k}, \Omega^{* k}\right], k=1: K$. By instead choosing to separate them into $K$ series of $L$ sample paths, we mitigate the risk of an unusual sample path that disproportionately favors a select group of control vectors.

\section{System Performance LOWER Bound}

In order to properly assess the performance of the parametric controllers developed, we establish in what follows a lower bound on best performance possible on average (i.e., it is possible to construct a sample path that performs better such as one without any $\delta_{i, j}$ request events)with an objective function equal to the absolute lower bound of zero). As the objective function in $[5$ is an average over $[0, T]$, we seek only an average performance lower bound.

Let us abstract the arrival process of discrete request events at rate $\lambda_{i, j}$ into a continuous request flow process with rate $\lambda_{i, j}$. On average, region $i$ will have an inflow of $\sum_{j=1}^{N} \lambda_{j, i}$ and an outflow of $\sum_{j=1}^{N} \lambda_{i, j}$. The average difference in request flow is given by: $d_{i}=\sum_{j=1}^{N}\left(\lambda_{i, j}-\lambda_{j, i}\right)$ such that we may define the following two sets of regions depending on the sign of $d_{i}$ :

$$
\mathcal{G}=\left\{i \in \mathcal{N} \mid d_{i}<0\right\} \quad \mathcal{B}=\left\{i \in \mathcal{N} \mid d_{i} \geq 0\right\}
$$

Based on the objective function defined in (5), there are two sources of system costs: unsated user requests for ATs and empty AT traveling time. Regions within $\mathcal{B}$ will run out of AT flow as they are a more popular origin than destination, thus they will effectively be forced to reject a request flow $d_{i}$.

For this abstracted flow AT system an amount $p$ of flow lost by not being sated with available ATs costs the system $c(p)$ as defined by:

$$
c(p)=p \cdot \frac{1}{\sum_{i=1}^{N} \sum_{j=1}^{N} \lambda_{i, j}}
$$

Regions within $\mathcal{B}$ will build up excess AT flow and are candidates to send empty AT flow out. Suppose that $p$ empty ATs are sent from $i$ to $j$ at the beginning of a time period, with an average trip time $\frac{1}{\mu_{i, j}}$ for a total mean empty AT driving time $\frac{p}{\mu_{i, j}}$. As there are a total of $m$ AT-hours, the mean number of empty ATs driving is $\frac{p}{\mu_{i, j} m}$. Similarly, for the abstracted fluid AT system forcing an AT flow of $p$ from $i$ to $j$, the system cost incurred is as follows; Note that both (30) and 31) are linear functions of the flow $p$.

$$
C(p, i, j)=p \cdot \frac{1}{\mu_{i, j} m}
$$

Consider two types of decision variables: $\beta_{j}, j \in \mathcal{B}$, as the fraction of positive request difference $d_{j}$ that will be left unsated and $v_{i, j}$ as the forced empty AT flow from $i \in \mathcal{G}$ to $j \in \mathcal{B}$ that will sate the remaining $\left[1-\beta_{j}\right]$ fractional difference in flow. The following linear program finds a lower bound with less than $N^{2}+N$ decision variables. The first and second parts of the objective function are the cost of ignoring a fraction $\beta_{j}$ and sating the fraction $\left[1-\beta_{j}\right]$ of difference in request $d_{j}$ from (30) and 31, respectively. The first constraint requires that the fraction $\left[1-\beta_{j}\right]$ of difference in request is sated in "bad" regions. The second constraint places limitations on the empty AT flows from "good" regions.

$$
\begin{aligned}
\mathrm{LB}=\min _{v_{i, j}, \beta_{j}} \sum_{j \in \mathcal{B}}\left(\frac{d_{j} \beta_{j}}{\sum_{i=1}^{N} \sum_{k=1}^{N} \lambda_{i, k}}+\sum_{i \in \mathcal{G}} \frac{v_{i, j}}{\mu_{i, j} m}\right) & \\
\text { s. t. } \quad d_{j}\left(1-\beta_{j}\right) & =\sum_{i \in \mathcal{G}} v_{i, j} \\
& -d_{i} \geq \sum_{j \in \mathcal{B}} v_{i, j} \\
0 & \leq v_{i, j} \\
0 & \leq B_{j} \leq 1
\end{aligned} \quad i \in \mathcal{B}
$$

\section{Simulation Example: A 6-Region System}

Consider an $N=6 \mathrm{MoD}$ system with request and travel rates as shown in Table $[\mathrm{II}$ and an objective function weight $w=0.5$. In order to assess the performance of our eventdriven parametric controller, we compare it to the time-driven controller, the static controller in [9], the lower bound derived in 32 , and the case of no control whatsoever.

TABLE II

$N=6$ System REQUEST RATES $\lambda$ AND TRAVEL RATES $\mu$

\begin{tabular}{|c|c|c|c|c|c|}
\multicolumn{8}{c|}{$\lambda$ (demands/min) } \\
\hline 6 & 15 & 6 & 6 & 9 & 3 \\
\hline 3 & 6 & 3 & 6 & 6 & 12 \\
\hline 0 & 9 & 3 & 0 & 3 & 3 \\
\hline 6 & 3 & 0 & 6 & 3 & 0 \\
\hline 6 & 12 & 6 & 0 & 3 & 0 \\
\hline 6 & 18 & 3 & 3 & 6 & 6 \\
\hline
\end{tabular}

\begin{tabular}{|c|c|c|c|c|c|}
\multicolumn{7}{|c}{$\mu(1 / \mathrm{min})$} \\
\hline 12 & 9.6 & 4.8 & 8.4 & 2.4 & 3.6 \\
\hline 9.6 & 12 & 7.2 & 6 & 3.6 & 4.8 \\
\hline 4.8 & 7.2 & 12 & 4.8 & 3.6 & 9.6 \\
\hline 8.4 & 6 & 4.8 & 12 & 2.4 & 2.4 \\
\hline 2.4 & 3.6 & 3.6 & 2.4 & 12 & 8.4 \\
\hline 3.6 & 4.8 & 9.6 & 2.4 & 8.4 & 12 \\
\hline
\end{tabular}

The event-driven and time-driven controllers require $N+1$ integer and 1 real-valued parameters respectively, which are determined using the concurrent estimation techniques described in section $\mathrm{V}$. After running many iterations of the greedy iteration as described by Section $\mathrm{V}-\mathrm{B}$ on a shared cloud computer cluster in MATLAB 2018b, the event and time-driven parameters in Table III were determined to perform the best for fleet sizes of 50, 75, 100, and 125 . 
TABLE III

Event And Time-Driven Controller Parameters: $N=6$ System

\begin{tabular}{|c|c|c|c|c|c|c|c|c|}
\hline Control & \multicolumn{7}{|c|}{ Event-Driven } & Time-Driven \\
\hline$m$ & $\theta_{1}$ & $\theta_{2}$ & $\theta_{3}$ & $\theta_{4}$ & $\theta_{5}$ & $\theta_{6}$ & $\Omega$ & $\Omega$ \\
\hline 50 & 10 & 7 & 4 & 1 & 4 & 7 & 5 & 24 \\
\hline 75 & 15 & 13 & 8 & 4 & 12 & 13 & 8 & 12 \\
\hline 100 & 20 & 16 & 11 & 7 & 16 & 20 & 14 & 12 \\
\hline 125 & 27 & 19 & 13 & 7 & 19 & 25 & 22 & 18 \\
\hline
\end{tabular}

TABLE IV

RANDOM SEARCH ITERATION-SPECIFIC PARAMETERS

\begin{tabular}{|c|c|c|c|c|c|}
\hline Iteration & $1-3$ & $4-6$ & $7-9$ & $10-12$ & $13-15$ \\
\hline$T$ & 500 & 5,000 & \multicolumn{3}{|c|}{10,000} \\
\hline$L$ & 25 & 50 & 100 & 200 & 500 \\
\hline$K$ & \multicolumn{5}{|c|}{25} \\
\hline
\end{tabular}

As these parameter vectors were determined to be the best via simulation, they are likely local minima of the objective function. Iterations stopped when slight deviations (i.e. $\theta_{i}^{\prime}=\theta_{i}+1$ ) had little effect on the objective function of a sufficiently long sample path ( $T=100,000$ time units).

Likewise, families of control vectors were found using the alternate random search method of finding well performing vectors as described in Section $\mathrm{V}-\mathrm{C}$ for each fleet size of [50,75,100,125] with iteration-specific $T, L$, and $K$ :

Table $\mathrm{V}$ shows the final well performing families of control vectors from two independent trials of 15 successive iterations. Note that these independent trials have very similar final control vector families and include the control vectors found via iterative greedy search as in Table III]

The static controller introduced in [9] requires $N^{2}$ parameters found via the LP (15) and shown in Table VI

Figure 3 shows the average simulated performance for all fleet sizes and controllers. All systems performed about the same under no control - over $37 \%$ of user requests unsated. As expected, the $N+1$ parameter event-driven controller with its state-dependent control and system-specific tuned parameters performs the best across all fleet sizes.

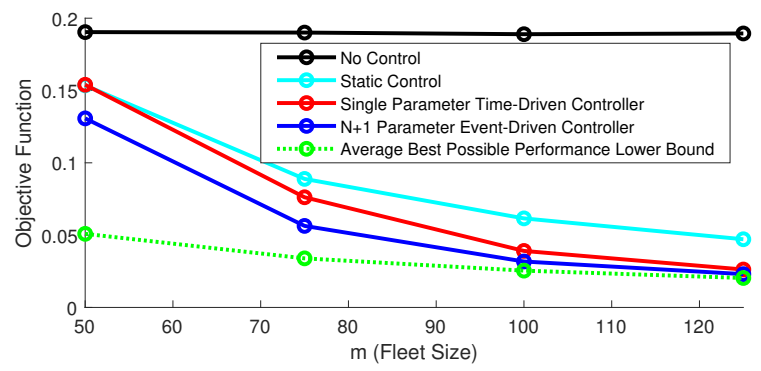

Fig. 3. The performance of a system with fleet sizes 50, 75, 100, and 125 under no control, static control, time-driven control, event-driven control, and the average best possible performance lower bound.

The intermediate fleet sizes studied here are where the true benefits of the event-driven controller are to be observed for this particular 6-region system. Note that the event and timedriven controllers quickly approach the lower bound as the fleet size increases but the static controller does not- this advantage is due to the event and time-driven controllers use of state information. We ignore fleets of less than 50: they are unstable and perform poorly no matter the control, since the underlying MoD system is under-capacitated with an insufficient number of vehicles to satisfy the given demand. We also ignore fleets over 125: they perform well no matter the control, as they are over-capacitated.

Table VII shows a detailed performance comparison of the three controllers relative to the uncontrolled case and the lower bound derived in 32 for a fleet size of 75 ATs.

Table VIII] a complement to Table [. shows the range of percents of fictitious events created by the Standard Clock method and the variation thereof in Section $\nabla$ for a fleet size of $m=75$ ATs. The variation of the Standard Clock method avoids many more fictitious events - enough to justify the extra calculations required.

\section{CONCLUSIONS AND FUture WORK}

MoD systems must load balance by sending empty vehicles to mitigate the temporal demand patterns that deplete some service regions of available vehicles. We have defined an objective function to jointly minimize the fraction of user requests denied due to unavailability and the fraction of time vehicles drive empty- and derived its lower bound. As optimal control via DP quickly becomes intractable even for small dimensionality systems, we have developed a parametric controller using thresholds on the number of vehicles available in and en route to each region. Optimal (or at least well-performing) parameters were determined using Concurrent Estimation methods which allow for the construction of multiple sample paths under different parameters from a single nominal sample path. Our simulation examples for the proposed event-driven threshold-based controller perform significantly better than an uncontrolled system or static controller and approach the lower bound for large fleet sizes.

Future work will include using actual taxi data and exploring a wider range of the control parameter space by making a more efficient use of $\mathrm{CE}$ methods through which we may attain a global optimum.

\section{REFERENCES}

[1] J. B. Greenblatt and S. Saxena, "Autonomous taxis could greatly reduce greenhouse-gas emissions of us light-duty vehicles," Nature Climate Change, vol. 5, no. 9, pp. 860-863, 2015.

[2] R. Zhang and M. Pavone, "Control of robotic mobility-on-demand systems: a queueing-theoretical perspective," The International Journal of Robotics Research, vol. 35, no. 1-3, pp. 186-203, 2016.

[3] W. Burghout, P. J. Rigole, and I. Andreasson, "Impacts of shared autonomous taxis in a metropolitan area," in Proceedings of the 94th Annual Meeting of the Transportation Research Board, 2015.

[4] C. Brownell and A. Kornhauser, "A driverless alternative: fleet size and cost requirements for a statewide autonomous taxi network in new jersey," Transportation Research Record: Journal of the Transportation Research Board, no. 2416, pp. 73-81, 2014.

[5] K. Spieser, K. Treleaven, R. Zhang, E. Frazzoli, D. Morton, and M. Pavone, "Toward a systematic approach to the design and evaluation of automated mobility-on-demand systems: A case study in singapore," in Road Vehicle Automation. Springer, 2014, pp. 229245.

[6] K. Spieser, S. Samaranayake, and E. Frazzoli, "Vehicle routing for shared-mobility systems with time-varying demand," in American Control Conference (ACC), 2016. IEEE, 2016, pp. 796-802. 
TABLE V

FAMilies of Well PERForming Vectors: Iterative RANDOM SEARCH

\begin{tabular}{|c|c|c|c|c|c|c|c|}
\hline$m$ & {$\left[\theta_{1}, \overline{\theta_{1}}\right]$} & {$\left[\theta_{2}, \overline{\theta_{2}}\right]$} & {$\left[\theta_{3}, \overline{\theta_{3}}\right]$} & {$\left[\theta_{4}, \overline{\theta_{4}}\right]$} & {$\left[\theta_{5}, \overline{\theta_{5}}\right]$} & {$\left[\theta_{6}, \overline{\theta_{6}}\right]$} & {$[\underline{\Omega}, \bar{\Omega}]$} \\
\hline \multirow{2}{*}{50} & {$[9,12]$} & {$[4,9]$} & {$[2,5]$} & {$[0,3]$} & {$[1,5]$} & {$[6,8]$} & {$[4,8]$} \\
\cline { 2 - 8 } & {$[9,12]$} & {$[1,9]$} & {$[2,5]$} & {$[0,3]$} & {$[0,5]$} & {$[6,8]$} & {$[3,8]$} \\
\hline \multirow{2}{*}{75} & {$[15,16]$} & {$[12,14]$} & {$[7,8]$} & {$[2,5]$} & {$[10,13]$} & {$[13,15]$} & {$[8,11]$} \\
\cline { 2 - 8 } & {$[14,17]$} & {$[11,14]$} & {$[7,9]$} & {$[0,6]$} & {$[10,13]$} & {$[12,15]$} & {$[6,11]$} \\
\hline \multirow{2}{*}{100} & {$[19,23]$} & {$[15,17]$} & {$[9,12]$} & {$[4,8]$} & {$[14,18]$} & {$[18,21]$} & {$[12,17]$} \\
\cline { 2 - 8 } & {$[18,22]$} & {$[15,18]$} & {$[9,12]$} & {$[1,8]$} & {$[14,17]$} & {$[17,22]$} & {$[10,16]$} \\
\hline \multirow{2}{*}{125} & {$[24,30]$} & {$[17,21]$} & {$[11,15]$} & {$[5,12]$} & {$[18,22]$} & {$[22,28]$} & {$[16,25]$} \\
\cline { 2 - 8 } & {$[24,30]$} & {$[16,22]$} & {$[11,16]$} & {$[2,11]$} & {$[17,21]$} & {$[23,28]$} & {$[16,26]$} \\
\hline
\end{tabular}

TABLE VI

Static Controller Parameters for the $N=6$ System

\begin{tabular}{|c|c|c|c|c|c|c|c|}
\multicolumn{2}{c}{} & \multicolumn{8}{c|}{ Destination } \\
\cline { 2 - 8 } & $r_{i, j}$ & 1 & 2 & 3 & 4 & 5 & 6 \\
\hline \multirow{4}{*}{ Origin } & 1 & 0 & 0 & 0 & 0 & 0 & 0 \\
\cline { 2 - 8 } & 2 & 0.25 & 0 & 0 & 0 & 0 & 0.2 \\
\cline { 2 - 8 } & 3 & 0 & 0 & 0 & 0 & 0 & 0.05 \\
\cline { 2 - 8 } & 4 & 0.5 & 0 & 0 & 0 & 0 & 0 \\
\cline { 2 - 8 } & 5 & 0 & 0 & 0 & 0 & 0 & 0.05 \\
\cline { 2 - 8 } & 6 & 0 & 0 & 0 & 0 & 0 & 0 \\
\hline
\end{tabular}

TABLE VII

$N=6, m=75$ SYSTEM PERFORMANCE COMPARISON

\begin{tabular}{|c|c|c|c|c|c|}
\hline Control & None & Static & $\begin{array}{c}\text { Time } \\
\text { Driven }\end{array}$ & $\begin{array}{c}\text { Event } \\
\text { Driven }\end{array}$ & $\begin{array}{c}\text { Lower } \\
\text { Bound }\end{array}$ \\
\hline$\%$ Users Rejected & 38.0 & 11.8 & 7.0 & 3.4 & 0 \\
\hline$\%$ ATs Drive Empty & 0 & 6.0 & 8.2 & 7.8 & 6.8 \\
\hline $\bar{J}(w=0.5)$ & 19.0 & 8.9 & 7.6 & 5.6 & 3.4 \\
\hline
\end{tabular}

[7] M. Ramezani and M. Nourinejad, "Dynamic modeling and control of taxi services in large-scale urban networks: A macroscopic approach," Transportation Research Part C: Emerging Technologies, vol. 94, pp. 203-219, 2018.

[8] D. J. Fagnant and K. M. Kockelman, "The travel and environmental implications of shared autonomous vehicles, using agent-based model scenarios," Transportation Research Part C: Emerging Technologies, vol. 40, pp. 1-13, 2014.

[9] M. Pavone, S. L. Smith, E. Frazzoli, and D. Rus, "Robotic load balancing for mobility-on-demand systems," The International Journal of Robotics Research, vol. 31, no. 7, pp. 839-854, 2012.

[10] J. Alonso-Mora, S. Samaranayake, A. Wallar, E. Frazzoli, and D. Rus, "On-demand high-capacity ride-sharing via dynamic trip-vehicle assignment," Proceedings of the National Academy of Sciences, vol. 114, no. 3, pp. 462-467, 2017.

[11] M. Vazifeh, P. Santi, G. Resta, S. Strogatz, and C. Ratti, "Addressing the minimum fleet problem in on-demand urban mobility," Nature, vol. 557, no. 7706, p. 534, 2018.

[12] C. G. Cassandras and C. G. Panayiotou, "Concurrent sample path analysis of discrete event systems," Discrete Event Dynamic Systems, vol. 9, no. 2, pp. 171-195, 1999.

[13] C. G. Cassandras and S. Lafortune, Introduction to Discrete Event Systems. Springer Science \& Business Media, 2009.

[14] D. P. Bertsekas, Dynamic programming and optimal control. Athena scientific Belmont, MA, 2005, vol. 1, no. 3.

[15] M. S. Bazaraa, Linear programming and network flows, fourth edition.. ed. Hoboken, New Jersey: Wiley, 2010.

\section{Appendix A: Optimal Control Policies of Simple SYSTEMS}

The optimal control policies for simple systems are possible to determine because they have tractable steady-state distributions. For a simple 2-region 1-AT time-invariant system (omit $k$ ) there are only eight possible states: the
TABLE VIII

Percent Fictitious Events for Fleet Size $m=75$

\begin{tabular}{|l|c|c|}
\cline { 2 - 3 } \multicolumn{1}{c|}{} & Minimum & Maximum \\
\hline Standard Clock & 93.91 & 98.96 \\
\hline Variation & 0 & 82.87 \\
\hline
\end{tabular}

single AT is either in one of the two idle AT queues $\mathcal{N}_{i}$, full with a passenger in server $W_{i, j}$ (including $i=j$ ), or empty in server $W_{i, j}$ (excluding the obviously undesirable empty intra-region trips $i=j$ ) as listed in Table IX] Let the user

TABLE IX

States OF THE TIME-INVARIANT $N=2, m=1$ SyStem

\begin{tabular}{|c|c|c|c|c|c|c|}
\hline State & $x_{1}$ & $x_{2}$ & $\begin{array}{l}y_{1,1} \\
y_{2,1}\end{array}$ & $\begin{array}{l}y_{1,2} \\
y_{2,2}\end{array}$ & $\begin{array}{l}z_{1,1} \\
z_{2,1}\end{array}$ & $\begin{array}{l}z_{1} \\
z_{2}\end{array}$ \\
\hline$N_{1}$ & {$[1$} & 0 & $\begin{array}{l}0 \\
0\end{array}$ & $\begin{array}{l}0 \\
0\end{array}$ & $\begin{array}{l}0 \\
0\end{array}$ & $\begin{array}{l}0 \\
0\end{array}$ \\
\hline$N_{2}$ & 0 & 1 & 0 & $\begin{array}{l}0 \\
0\end{array}$ & $\begin{array}{l}0 \\
0\end{array}$ & $\begin{array}{l}0 \\
0\end{array}$ \\
\hline$W_{1,1}$ & 0 & 0 & 1 & $\begin{array}{l}0 \\
0\end{array}$ & $\begin{array}{l}0 \\
0\end{array}$ & $\begin{array}{l}0 \\
0\end{array}$ \\
\hline$W_{1,2}$ & 0 & 0 & 0 & $\begin{array}{l}1 \\
0\end{array}$ & $\begin{array}{l}0 \\
0\end{array}$ & $\begin{array}{l}0 \\
0\end{array}$ \\
\hline$W_{2,1}$ & 0 & 0 & $\begin{array}{l}0 \\
1\end{array}$ & $\begin{array}{l}0 \\
0\end{array}$ & $\begin{array}{l}0 \\
0\end{array}$ & $\begin{array}{l}0 \\
0\end{array}$ \\
\hline$W_{2,2}$ & {$[0$} & 0 & 0 & $\begin{array}{l}0 \\
1\end{array}$ & $\begin{array}{l}0 \\
0\end{array}$ & $\begin{array}{l}0 \\
0\end{array}$ \\
\hline$W_{1,2}^{E}$ & 0 & 0 & 0 & $\begin{array}{l}0 \\
0\end{array}$ & $\begin{array}{l}0 \\
0\end{array}$ & $\begin{array}{l}1 \\
0\end{array}$ \\
\hline$W_{2,1}^{E}$ & {$[0$} & 0 & 0 & $\begin{array}{l}0 \\
0\end{array}$ & $\begin{array}{l}0 \\
1\end{array}$ & $\begin{array}{l}0 \\
0\end{array}$ \\
\hline
\end{tabular}

arrivals follow a Poisson process with rate $\lambda_{i}$ and infinitecapacity server $W_{i, j}$ be exponential with mean service time $\frac{1}{\mu_{i, j}}$. The simple $N=2, m=1$ system without controls (omit empty driving states $W_{i, j}^{E}$ ) may now be represented by the continuous Markov chain in Figure 4 . For simplicity of notation, let $\lambda_{i, j}=p_{i, j} \lambda_{i}$, the rate at which users arrive to region $i$ destined for region $j$. Instead of individual AT controls $u_{i, j, q}$, consider a controllable state transition rate $\beta_{i, j} \in[0, \infty)$ as the rate at which to send an empty AT from region $i$ to region $j$ [2],[5]. This controllable Markov Chain for the $N=2, m=1$ system in Figure 5 includes empty AT states $W_{1,2}^{E}$ and $W_{2,1}^{E}$. We may calculate the steady-state probability vector $\pi_{\beta}$ from the normalized transition matrix with rates from Figure 5 where $\beta=\left[\beta_{1,2}, \beta_{2,1}\right]$. The cost 


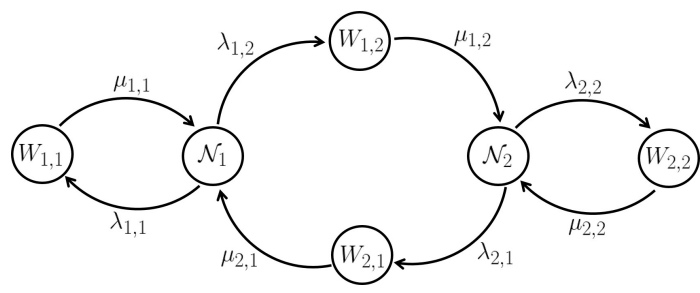

Fig. 4. The simplest possible system, the time-invariant 2-region 1-AT system sans controls has six possible states: the AT may be in either of the idle AT queues $\mathcal{N}_{i}$ or in one of the four servers $W_{i, j}$; the system moves between these six states at rates $\mu_{i, j}$ and $\lambda_{i, j}$ where $\lambda_{i, j}=p_{i, j} \lambda_{i}$.

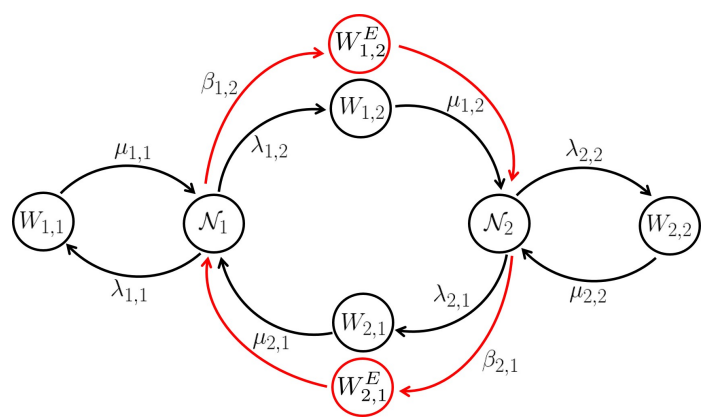

Fig. 5. The time-invariant $N=2, m=1$ system with controllable transition rates $\beta_{1,2}$ and $\beta_{2,1}$ as the rates at which empty ATs are sent from 1 to 2 and 2 to 1 , respectively, leads to an 8 state system with the addition of empty AT states $W_{1,2}^{E}$ and $W_{2,1}^{E}$.

$C(\mathscr{X})$ associated with being in state $\mathscr{X}$ is as follows:

$$
C(\mathscr{X})=w \sum_{i=1}^{N} \frac{\sum_{j} \lambda_{i, j} \mathbf{1}\left[x_{i}=0\right]}{\sum_{i} \sum_{j}\left(\lambda_{i, j}+\mu_{i, j}\right)}+(1-w) \frac{\sum_{i} \sum_{j} z_{i, j}}{m}
$$

The full objective function of this $N=2, m=1$ system may be written in terms of the probabilities:

$$
\begin{array}{r}
J^{*}=\min _{\boldsymbol{\beta}}\left\{w \frac{\left(\lambda_{1,1}+\lambda_{1,2}\right)\left(1-\pi_{\boldsymbol{\beta}}\left(\mathcal{N}_{1}\right)\right)+\left(\lambda_{2,1}+\lambda_{2,2}\right)\left(1-\pi_{\boldsymbol{\beta}}\left(\mathcal{N}_{2}\right)\right)}{\sum_{i} \sum_{j} \lambda_{i, j}}\right. \\
\left.+(1-w)\left(\pi_{\boldsymbol{\beta}}\left(W_{1,2}^{E}\right)+\pi_{\boldsymbol{\beta}}\left(W_{2,1}^{E}\right)\right)\right\}
\end{array}
$$

Consider the simplest scenario in which the percents of rejected requests and load balancing vehicles are equally weighted with $w=0.5$ such that we may ignore $w$. The objective function may be alternatively shown to be a ratio of polynomial functions of the $\beta_{1,2}$ and $\beta_{2,1}$ control rates:

$$
f\left(\beta_{1,2}, \beta_{2,1}\right)=\frac{A \beta_{1,2} \beta_{2,1}+B \beta_{1,2}+C \beta_{2,1}+D}{E \beta_{1,2} \beta_{2,1}+F \beta_{1,2}+G \beta_{2,1}+H}
$$

where $A$ through $H$ are all positive complicated combinations of $\lambda_{i, j}$ and $\mu_{i, j}$ listed in Appendix B.

Inspection of $\mathrm{A}$ and $\mathrm{E}$ in Appendix $\mathrm{B}$ reveals that $A=2 E$ such that the objective function can be rewritten as:

$$
f\left(\beta_{1,2}, \beta_{2,1}\right)=\frac{A \beta_{1,2} \beta_{2,1}+B \beta_{1,2}+C \beta_{2,1}+D}{0.5 A \beta_{1,2} \beta_{2,1}+F \beta_{1,2}+G \beta_{2,1}+H}
$$

As both $\beta_{1,2}$ and $\beta_{2,1} \rightarrow \infty$ the objective function goes to its upper bound of 2 as the AT cycles empty between the two regions and never serves any users:

$$
\lim _{\beta_{1,2} \cdot \beta_{2,1} \rightarrow \infty} f\left(\beta_{1,2}, \beta_{2,1}\right)=\frac{A}{.5 A}=2
$$

leading to $100 \%$ of user requests missed and $100 \%$ of AT timing driving empty.

The derivative of the objective function with regard to $\beta_{1,2}$

$$
\begin{aligned}
& \quad \frac{\partial f\left(\beta_{1,2}, \beta_{2,1}\right)}{\partial \beta_{1,2}}= \\
& \frac{A \beta_{2,1}\left(G \beta_{2,1}+H\right)+B\left(G \beta_{2,1}+H\right)+\left(C \beta_{2,1}+D\right)\left(-E \beta_{2,1}-F\right)}{\left(H+F \beta_{1,2}+G \beta_{2,1}+E \beta_{1,2} \beta_{2,1}\right)^{2}}
\end{aligned}
$$

has an either always positive or negative numerator which is not a function of $\beta_{1,2}$ and a denominator that is an always positive function of $\beta_{1,2}$. Hence $\frac{\partial f\left(\beta_{1,2}, \beta_{2,1}\right)}{\partial \beta_{1,2}}$ is either always positive or always negative such that the $\beta_{1,2}$ to minimize the objective function will be found on the boundaries 0 or $\infty$.

Fixing $\beta_{2,1}$ to consider the objective as a function of $\beta_{1,2}$ :

$$
f\left(\beta_{1,2}\right)=\frac{\left(A \beta_{2,1}+B\right) \beta_{1,2}+\left(C \beta_{2,1}+D\right)}{\left(E \beta_{2,1}+F\right) \beta_{1,2}+\left(G \beta_{2,1}+H\right)}
$$

we may regroup: $a=A \beta_{2,1}+B, b=C \beta_{2,1}+D, c=$ $E \beta_{2,1}+F, d=G \beta_{2,1}+H$ with $a, b, c, d \geq 0$ to rewrite the objective function as:

$$
f\left(\beta_{1,2}\right)=\frac{a \beta_{1,2}+b}{c \beta_{1,2}+d}
$$

where the first and second derivatives are:

$$
\begin{gathered}
\frac{d f\left(\beta_{1,2}\right)}{d \beta_{1,2}}=\frac{a d-c b}{\left(c \beta_{1,2}+d\right)^{2}} \\
\frac{d^{2} f\left(\beta_{1,2}\right)}{d \beta_{1,2}{ }^{2}}=\frac{-2 c\left(c \beta_{1,2}+d\right)(a d-c b)}{\left(c \beta_{1,2}+d\right)^{4}}
\end{gathered}
$$

If we assume $a d>c b$ then the first derivative is always positive meaning the objective function is monotonically increasing and therefore minimized at $\beta_{1,2}=0$ for $f(0)=\frac{b}{d}$. Furthermore the second derivative will always be negative implying the objective function is concave. Likewise if we assume $a d<c b$ then the first derivative is always negative meaning the objective function monotonically decreasing and is minimized at $\beta_{1,2}=\infty$ for $f(\infty)=\frac{a}{c}$. The second derivative is always positive and implies the objective function is convex. In the same way by fixing $\beta_{1,2}$, we may show that the optimal choice of $\beta_{2,1}$ is either at 0 or $\infty$.

As the optimal choices for $\beta_{1,2}$ and $\beta_{2,1}$ are both either 0 or $\infty$ there are only four possible sets of optimal controls and a simple comparison rule to determine the optimal policy:

$$
\left(\beta_{1,2}, \beta_{2,1}\right)= \begin{cases}(\infty, \infty) & \text { if } \min \left\{\frac{A}{E}, \frac{B}{F}, \frac{C}{G}, \frac{D}{H}\right\}=\frac{A}{E} \\ (\infty, 0) & \text { if } \min \left\{\frac{A}{E}, \frac{B}{F}, \frac{C}{G}, \frac{D}{H}\right\}=\frac{B}{F} \\ (0, \infty) & \text { if } \min \left\{\frac{A}{E}, \frac{B}{F}, \frac{C}{G}, \frac{D}{H}\right\}=\frac{C}{G} \\ (0,0) & \text { if } \min \left\{\frac{A}{E}, \frac{B}{F}, \frac{C}{G}, \frac{D}{H}\right\}=\frac{D}{H}\end{cases}
$$

As previously noted, the first case will never occur as this would send the objective function to its upper bound of 2 . 
Appendix B: Optimal Policy fOR a $N=2, m=1$ EQUAlly Weighted $w=0.5$ System Using RAte $\beta_{i, j}$

$$
A=2\left(\mu_{11} \mu_{12} \mu_{22}+\mu_{11} \mu_{21} \mu_{22}\right)\left(\lambda_{11}+\lambda_{12}+\lambda_{21}+\lambda_{22}\right)
$$

$\lambda_{21}^{2} \mu_{11} \mu_{12} \mu_{22}+\lambda_{22}^{2} \mu_{11} \mu_{12} \mu_{21}$

$+2 \lambda_{21}^{2} \mu_{11} \mu_{21} \mu_{22}+\lambda_{11} \lambda_{21} \mu_{11} \mu_{12} \mu_{22}$

$+\lambda_{11} \lambda_{22} \mu_{11} \mu_{12} \mu_{21}+\lambda_{12} \lambda_{21} \mu_{11} \mu_{12} \mu_{22}$

$B=+\lambda_{12} \lambda_{22} \mu_{11} \mu_{12} \mu_{21}+2 \lambda_{11} \lambda_{21} \mu_{11} \mu_{21} \mu_{22}$

$+2 \lambda_{12} \lambda_{21} \mu_{11} \mu_{21} \mu_{22}+\lambda_{21} \lambda_{22} \mu_{11} \mu_{12} \mu_{21}$

$+\lambda_{21} \lambda_{22} \mu_{11} \mu_{12} \mu_{22}+2 \lambda_{21} \lambda_{22} \mu_{11} \mu_{21} \mu_{22}$

$+\lambda_{11} \mu_{11} \mu_{12} \mu_{21} \mu_{22}+\lambda_{12} \mu_{11} \mu_{12} \mu_{21} \mu_{22}$

$2 \lambda_{12}^{2} \mu_{11} \mu_{12} \mu_{22}+\lambda_{11}^{2} \mu_{12} \mu_{21} \mu_{22}$ $+\lambda_{12}^{2} \mu_{11} \mu_{21} \mu_{22}+2 \lambda_{11} \lambda_{12} \mu_{11} \mu_{12} \mu_{22}$

$+\lambda_{11} \lambda_{12} \mu_{11} \mu_{21} \mu_{22}+\lambda_{11} \lambda_{12} \mu_{12} \mu_{21} \mu_{22}$

$C=+2 \lambda_{12} \lambda_{21} \mu_{11} \mu_{12} \mu_{22}+2 \lambda_{12} \lambda_{22} \mu_{11} \mu_{12} \mu_{22}$

$+\lambda_{11} \lambda_{21} \mu_{12} \mu_{21} \mu_{22}+\lambda_{12} \lambda_{21} \mu_{11} \mu_{21} \mu_{22}$

$+\lambda_{11} \lambda_{22} \mu_{12} \mu_{21} \mu_{22}+\lambda_{12} \lambda_{22} \mu_{11} \mu_{21} \mu_{22}$

$+\lambda_{21} \mu_{11} \mu_{12} \mu_{21} \mu_{22}+\lambda_{22} \mu_{11} \mu_{12} \mu_{21} \mu_{22}$

$\lambda_{12} \lambda_{21}^{2} \mu_{11} \mu_{12} \mu_{22}+\lambda_{12} \lambda_{22}{ }^{2} \mu_{11} \mu_{12} \mu_{21}$

$+\lambda_{12}{ }^{2} \lambda_{21} \mu_{11} \mu_{12} \mu_{22}+\lambda_{12}{ }^{2} \lambda_{22} \mu_{11} \mu_{12} \mu_{21}$

$+\lambda_{11} \lambda_{21}{ }^{2} \mu_{12} \mu_{21} \mu_{22}+\lambda_{12} \lambda_{21}{ }^{2} \mu_{11} \mu_{21} \mu_{22}$

$+\lambda_{11}{ }^{2} \lambda_{21} \mu_{12} \mu_{21} \mu_{22}+\lambda_{12}{ }^{2} \lambda_{21} \mu_{11} \mu_{21} \mu_{22}$

$D=+\lambda_{12}{ }^{2} \mu_{11} \mu_{12} \mu_{21} \mu_{22}+\lambda_{21}^{2} \mu_{11} \mu_{12} \mu_{21} \mu_{22}$

$+\lambda_{11} \lambda_{12} \lambda_{21} \mu_{11} \mu_{12} \mu_{22}+\lambda_{11} \lambda_{12} \lambda_{22} \mu_{11} \mu_{12} \mu_{21}$

$+\lambda_{11} \lambda_{12} \lambda_{21} \mu_{11} \mu_{21} \mu_{22}+\lambda_{11} \lambda_{12} \lambda_{21} \mu_{12} \mu_{21} \mu_{22}$

$+\lambda_{12} \lambda_{21} \lambda_{22} \mu_{11} \mu_{12} \mu_{21}+\lambda_{12} \lambda_{21} \lambda_{22} \mu_{11} \mu_{12} \mu_{22}$

$+\lambda_{11} \lambda_{21} \lambda_{22} \mu_{12} \mu_{21} \mu_{22}+\lambda_{12} \lambda_{21} \lambda_{22} \mu_{11} \mu_{21} \mu_{22}$

$+\lambda_{11} \lambda_{12} \mu_{11} \mu_{12} \mu_{21} \mu_{22}+\lambda_{21} \lambda_{22} \mu_{11} \mu_{12} \mu_{21} \mu_{22}$

$E=\frac{A}{2}=\left(\mu_{11} \mu_{12} \mu_{22}+\mu_{11} \mu_{21} \mu_{22}\right)\left(\lambda_{11}+\lambda_{12}+\lambda_{21}+\lambda_{22}\right)$

$$
F=\left(\lambda_{11}+\lambda_{12}+\lambda_{21}+\lambda_{22}\right) *
$$

$\left(\lambda_{21} \mu_{11} \mu_{12} \mu_{22}+\lambda_{22} \mu_{11} \mu_{12} \mu_{21}+\right.$

$\left.\lambda_{21} \mu_{11} \mu_{21} \mu_{22}+\mu_{11} \mu_{12} \mu_{21} \mu_{22}\right)$

$$
\begin{aligned}
& G=\left(\lambda_{11} \lambda_{12}+\lambda_{21}+\lambda_{22}\right) * \\
& \left(\lambda_{12} \mu_{11} \mu_{12} \mu_{22}+\lambda_{11} \mu_{12} \mu_{21} \mu_{22}+\right. \\
& \left.\quad \lambda_{12} \mu_{11} \mu_{21} \mu_{22}+\mu_{11} \mu_{12} \mu_{21} \mu_{22}\right)
\end{aligned}
$$

$$
\begin{aligned}
H= & \left(\lambda_{11}+\lambda_{12}+\lambda_{21}+\lambda_{22}\right) * \\
& \left(\lambda_{12} \lambda_{21} \mu_{11} \mu_{12} \mu_{22}+\lambda_{12} \lambda_{22} \mu_{11} \mu_{12} \mu_{21}\right. \\
& +\lambda_{11} \lambda_{21} \mu_{12} \mu_{21} \mu_{22}+\lambda_{12} \lambda_{21} \mu_{11} \mu_{21} \mu_{22} \\
& \left.\quad+\lambda_{12} \mu_{11} \mu_{12} \mu_{21} \mu_{22}+\lambda_{21} \mu_{11} \mu_{12} \mu_{21} \mu_{22}\right)
\end{aligned}
$$

\section{ApPendix C: Optimal Policy Using Dynamic \\ PROGRAMMING FOR THE $N=2, m=1$ EQUALLY Weighted $w=0.5$ SYSTEM}

The $N=2 m=1$ equally weighed $w=0.5$ dynamic linear program may be written as:

$$
\begin{aligned}
& \max _{\bar{J}, h\left(\mathcal{N}_{1}\right), h\left(\mathcal{N}_{2}\right), h\left(W_{1,1}\right), h\left(W_{1,2}\right), h\left(W_{2,1}\right), h\left(W_{2,2}\right), h\left(W_{1,1}^{E}\right), h\left(W_{2,1}^{E}\right)}^{\bar{J}} \\
& \text { s. t. } \bar{J}+h\left(\mathcal{N}_{1}\right) \leq \frac{\sum_{j} \lambda_{2, j}}{\alpha}+\frac{\lambda_{1,1}}{\alpha} h\left(W_{1,1}\right) \\
& +\frac{\lambda_{1,2}}{\alpha} h\left(W_{1,2}\right)+\left(1-\frac{\sum_{j} \lambda_{1, j}}{\alpha}\right) h\left(\mathcal{N}_{1}\right) \\
& \bar{J}+h\left(\mathcal{N}_{2}\right) \leq \frac{\sum_{j} \lambda_{1, j}}{\alpha}+\frac{\lambda_{2,1}}{\alpha} h\left(W_{2,1}\right)+\frac{\lambda_{2,2}}{\alpha} h\left(W_{2,2}\right) \\
& +\left(1-\frac{\sum_{j} \lambda_{2, j}}{\alpha}\right) h\left(\mathcal{N}_{2}\right) \\
& \bar{J}+h\left(W_{1,1}\right) \leq 1+\frac{\mu_{1,1}}{\alpha} h\left(\mathcal{N}_{1}\right)+\left(1-\frac{\mu_{1,1}}{\alpha}\right) h\left(W_{1,1}\right) \\
& \bar{J}+h\left(W_{1,1}\right) \leq 1+\frac{\mu_{1,1}}{\alpha} h\left(W_{1,2}^{E}\right)+\left(1-\frac{\mu_{1,1}}{\alpha}\right) h\left(W_{1,1}\right) \\
& \bar{J}+h\left(W_{1,2}\right) \leq 1+\frac{\mu_{1,2}}{\alpha} h\left(\mathcal{N}_{2}\right)+\left(1-\frac{\mu_{1,2}}{\alpha}\right) h\left(W_{1,2}\right) \\
& \bar{J}+h\left(W_{1,2}\right) \leq 1+\frac{\mu_{1,2}}{\alpha} h\left(W_{2,1}^{E}\right)+\left(1-\frac{\mu_{1,2}}{\alpha}\right) h\left(W_{1,2}\right) \\
& \bar{J}+h\left(W_{2,1}\right) \leq 1+\frac{\mu_{2,1}}{\alpha} h\left(\mathcal{N}_{1}\right)+\left(1-\frac{\mu_{2,1}}{\alpha}\right) h\left(W_{2,1}\right) \\
& \bar{J}+h\left(W_{2,1}\right) \leq 1+\frac{\mu_{2,1}}{\alpha} h\left(W_{1,2}^{E}\right)+\left(1-\frac{\mu_{2,1}}{\alpha}\right) h\left(W_{2,1}\right) \\
& \bar{J}+h\left(W_{2,2}\right) \leq 1+\frac{\mu_{2,2}}{\alpha} h\left(\mathcal{N}_{2}\right)+\left(1-\frac{\mu_{2,2}}{\alpha}\right) h\left(W_{2,2}\right) \\
& \bar{J}+h\left(W_{2,2}\right) \leq 1+\frac{\mu_{2,2}}{\alpha} h\left(W_{2,1}^{E}\right)+\left(1-\frac{\mu_{2,2}}{\alpha}\right) h\left(W_{2,2}\right) \\
& \bar{J}+h\left(W_{1,2}^{E}\right) \leq 2+\frac{\mu_{1,2}}{\alpha} h\left(\mathcal{N}_{2}\right)+\left(1-\frac{\mu_{1,2}}{\alpha}\right) h\left(W_{1,2}^{E}\right) \\
& \bar{J}+h\left(W_{1,2}^{E}\right) \leq 2+\frac{\mu_{1,2}}{\alpha} h\left(W_{2,1}^{E}\right)+\left(1-\frac{\mu_{1,2}}{\alpha}\right) h\left(W_{1,2}^{E}\right) \\
& \bar{J}+h\left(W_{2,1}^{E}\right) \leq 2+\frac{\mu_{2,1}}{\alpha} h\left(\mathcal{N}_{1}\right)+\left(1-\frac{\mu_{2,1}}{\alpha}\right) h\left(W_{2,1}^{E}\right) \\
& \bar{J}+h\left(W_{2,1}^{E}\right) \leq 2+\frac{\mu_{2,1}}{\alpha} h\left(W_{1,2}^{E}\right)+\left(1-\frac{\mu_{2,1}}{\alpha}\right) h\left(W_{2,1}^{E}\right)
\end{aligned}
$$

with uniformized rate $\alpha \geq \sum_{i} \sum_{j} \lambda_{i, j}+\max \left\{\mu_{i, j}\right\}$ from Figure 4.

The first two constraints deal with the idle AT states of $\mathcal{N}_{1}$ and $\mathcal{N}_{2}$ which by this definition have no controls but have an expected cost associated with the ratio of users they expect to miss i.e. the \% which arrive at the other region. Constraints 3-10 are for the en routes states $W_{1,1}, W_{1,2}, W_{2,1}$, and $W_{2,2}$ each with 2 controls: do nothing and let the AT become idle or send the empty AT to the other region with an associated cost of 1 because $100 \%$ of expected user arrivals will be missed because the 1 and only AT is busy. Constraints 1114 deal with states $W_{1,2}^{E}$ and $W_{2,1}^{E}$ which echo states $W_{1,2}$ and $W_{2,1}$ in terms of controls but have an associated cost of 2 because $100 \%$ of users arriving will not obtain an AT and $100 \%$ of time in that state is an AT driving empty. 